\section{- CARDIOVASCULAR DISEASE}

\section{$T$ cell activation in} hypertension

Although studies in animal models of hypertension have demonstrated a key role for $T$ cells, it has been unclear how $T$ cells are activated under hypertensive conditions. A new study identifies protein oxidation by isoketals, which are derived from free radical-mediated lipid peroxidation of arachidonic acid, as contributing to $T$ cell activation and hypertension (J. Clin. Invest. 124, 4642-4656, 2014).

David Harrison and his colleagues found in two mouse models of hypertension-treatment with either angiotensin II or deoxycorticosterone plus $\mathrm{NaCl}$-that isoketal-modified proteins accumulated within splenic dendritic cells. For either model, treatment with an isoketal scavenger blunted the hypertensive response. Moreover, adoptive transfer of isoketal-laden dendritic cells sensitized the recipient mice to a low, subpressor dose of angiotensin $\mathrm{II}$-an effect that required the presence of $T$ cells in the recipient miceindicating the pathological role of this oxidized lipid in dendritic cells. Mechanistic studies showed that accumulation of isoketal-modified proteins in dendritic cells as a consequence of either oxidative stress within the cells or uptake of modified proteins led to increased cytokine production and $\mathrm{T}$ cell activation in coculture studies. The authors also showed that hypertensive patients have increased levels of plasma F2-isoprostanes, which are generated in the same pathway as isoketals, and of isoketal adducts in circulating mononuclear cells.

These findings link oxidative stress to the formation of neoepitopes that promote an autoimmune-like response, leading to hypertension. $-M B$

\section{DEPRESSION}

\section{Linking exercise and depression}

A study recently published in Ce/l has made a molecular link between exercise and resistance to depression (Cel/ 159, 33-45, 2014).

It is known that exercise induces expression of the transcriptional coactivator PGC-1a in muscle. Aguedlo et al. showed that mice in which PGC-1 $\alpha$ is overexpressed in muscle (mck-PGC1- $\alpha 1$ ) are resilient to developing depression-like behavior induced by chronic mild stress (CMS) and associated changes in neuroinflammation and synaptic proteins. Depression has been linked to the conversion of tryptophan to kynurenine. Mck-PGC1- $\alpha 1$ mice had increased expression of KAT enzymes, which convert kynurenine to kynurenic acid. These mice also had lower levels of kynurenine in response to CMS, suggesting that increased kynurenine metabolism mediates resilience in these animals. Furthermore, kynurenine administration in wild-type mice induced depression-like behavior. Mice lacking PGC1- $\alpha 1$ in muscle also had increased depression-like behavior and circulating kynurenine levels, even in the absence of CMS. The authors also showed in cell culture experiments that the stress-induced transcriptional activators PPAR- $\alpha$ and PPAR- $\delta$ are binding partners of PGC1- $\alpha$.

Endurance training in mice increased expression of PGC-1a and KAT enzymes in muscle, and increased plasma kynurenic acid levels. Furthermore, a 3-week training program in humans increased muscle expression of KAT enzymes, PPAR- $\alpha$ and PPAR- $\delta$. This suggests exercise-induced changes in kynurenine metabolism could also occur in humans, and so may explain the beneficial effects of exercise in depressed patients. - $-H S$

\section{IMMUNOLOGY \\ Myeloid cells mapped out}

An unbiased, high-dimensional characterization of the myeloid compartment using mass cytometry is described in the journal Nature Immunology (doi:10.1038/ni.3006, 2014).

Markers currently used to define myeloid cells are expressed by several cell types, and their expression may be altered by the inflammatory environment. Moreover, flow cytometry-based analyses are hampered by the number of parameters that can be analyzed and by overlap between channels. Mass cytometry makes use of heavy metal isotypes as tags, permitting analysis of many parameters at a single-cell level and discrimination between related cell types.

Burkhard Becher, Evan Newell and their colleagues used mass cytometry and a 38-antibody panel to analyze myeloid cells within lymphoid and nonlymphoid tissue under steady-state conditions. Using automated approaches, they identify several myeloid cell subsets, revealing the considerable phenotypic diversity among these cells. Although myeloid cells are heterogeneous and their phenotype differs on the basis of the resident tissue, most clusters were composed of cells from different tissues. However, brain microglia and lung alveolar macrophages formed a single cluster, whereas neutrophils were diverse. A comparison between Csf2rb- deficient and wild-type mice revealed a loss in alveolar macrophages, eosinophils and some dendritic cell populations in the Csf2rbdeficient mice, confirming prior reports, and an unexpected reduction in CD1 $1 b^{\text {lo }}$ natural killer cells.

These findings validate this unbiased approach to segregate and classify immune cell types and provide a useful resource for future studies. - KDS

\section{CANCER GENOMICS Mixed and matched tumors}

Two recent studies in Science try to delineate the variability and evolution of lung tumors.

Andrew Futreal and his colleagues (Science 346, 256-259, 2014) and Charles Swanton his colleagues (Science 346, 251-256, 2014) carried out whole-exome and wholegenome sequencing of multiple regions of 11 and 7 localized lung cancers, respectively, to depict their clonal composition. Both studies found divergent alterations between regions of the same tumor, suggesting branching evolution, but also that a high percentage of driver mutations are shared between regions. Variability seems to be driven by alterations in noncanonical cancer genes.

Futreal and colleagues showed that divergent mutations can be present in multiple regions but that there is intraregion allelic variability. Tumors that relapsed had a higher number of subclonal fractions, suggesting that although single biopsies could be appropriate to evaluate the genetic makeup of a tumor, the level of per-patient heterogeneity should be taken into account to predict progression. Swanton and colleagues corroborate that most driver and regional mutations seem to be clonal, but some regions show subclonal variability, warning about the potential "illusion of clonality" derived from sampling one tumor region.

Both studies suggest that smoking-derived mutational signatures drive early progression, whereas later evolution seems to bear the hallmarks of other mutational processes. Swanton and colleagues propose that events resulting in widespread genomic instability can take place before or after driver mutations appear.

Though the sample size is small, the studies provide a useful picture of the evolution landscape of lung tumors. - VA

Written by Victoria Aranda, Michael Basson, Kevin Da Silva \& Hannah Stower 